(2) Open Access Full Text Article

\title{
Preparation, characterization, and in vivo study of rhein-loaded poly(lactic-co-glycolic acid) nanoparticles for oral delivery
}

This article was published in the following Dove Press journal:

Drug Design, Development and Therapy

2I April 2015

Number of times this article has been viewed

\section{Zheng Yuan \\ Xinhua Gu}

Department of Gastrointestinal Surgery, Suzhou Municipal Hospital, Suzhou, People's Republic of China

Correspondence: Xinhua Gu Department of Gastrointestinal Surgery, Suzhou Municipal Hospital, No 26 Daoqian Street, Suzhou 215002, People's Republic of China Email xinhua_gu@yeah.net

\begin{abstract}
A novel rhein formulation based on poly(lactic-co-glycolic acid) (PLGA) nanoparticles (NPs) suitable for oral administration was developed in this study. The designed nanosystems were obtained by a modified spontaneous emulsification solvent diffusion method. The morphology of rhein-loaded PLGA NPs showed a spherical shape with a smooth surface, without any particle aggregation. Mean size of the NPs was $140.5 \pm 4.3 \mathrm{~nm}$, and the zeta potential was $-16.9 \pm 3.1 \mathrm{mV}$. The average drug loading was $3.9 \% \pm 0.7 \%$, and encapsulation efficiency was $84.5 \% \pm 6.2 \%$. Meanwhile, NPs are characterized by the slower release (only about $70 \%$ of rhein is released within 5 hours), and the model that fitted best for rhein released from the NPs was Higuchi kinetic model with correlation coefficient $r=0.9993$, revealing that rhein could be controlled released from the NPs. In vivo, NPs altered the distribution of rhein, and the half-life after oral administration was prolonged remarkably more than those of suspensions (22.6 hours vs 4.3 hours). The pharmacokinetic results indicated that the NPs had sustainedrelease efficacy. The area under the curve ${ }_{0-\infty}$ of the NPs formulation was 3.07-fold higher than that of suspensions, suggesting that the encapsulated rhein had almost been absorbed in rats over the period of 12 hours. Although rhein-loaded PLGA NP formulations are hopefully used as a chemotherapeutic or adjuvant agent for human gastric cancer (SGC-7901), their in vivo antitumor effect and mechanisms at the molecular level still need further study.
\end{abstract}

Keywords: rhein, PLGA, nanoparticles, release, pharmacokinetics, SGC-7901

\section{Introduction}

Rhein (4,5-dihydroxyanthraquinone-2-carboxylic acid; Figure 1), a primary anthraquinone present in the roots of rhubarb (Rheum palmatum L. or Rheum tanguticum Maxim.), is a traditional Chinese herbal medicine that has been used as a laxative and stomach drug for years. ${ }^{1,2}$ Recently, some in vivo studies found that rhein had the effect of inhibiting cell growth of liver tumors in rats, ${ }^{3}$ human glioma, ${ }^{4}$ and Ehrlich ascites tumor. ${ }^{5}$ At the same time, some basic research discovered that rhein can inhibit the growth of other cancer cells such as SCC-4 human tongue cancer cells, ${ }^{6-8} \mathrm{Caco}-2$ human adenocarcinoma cells, ${ }^{9}$ breast cancer cells, ${ }^{10}$ nasopharyngeal carcinoma cells, ${ }^{11}$ a-549 human lung cancer cells, ${ }^{12}$ bel-7402 human hepatoma cells, ${ }^{13}$ and Ca Ski human cervical cancer cells. ${ }^{14}$ However, since rhein is a hydrophobic drug, it would be very difficult for vascular route of administration. Thus, a new drug delivery system needed to be designed. For the problem of poor solubility of hydrophobic drug in water, the application of nanotechnology enabled researchers to see hope. ${ }^{15}$ Recently, a biodegradable polymer nanoparticle (NP) has shown its increasingly important academic value and significant clinical applications in cancer therapy. ${ }^{16,17}$ 
<smiles>O=C(O)c1cc(O)c2c(c1)C(=O)c1cccc(O)c1C2=O</smiles>

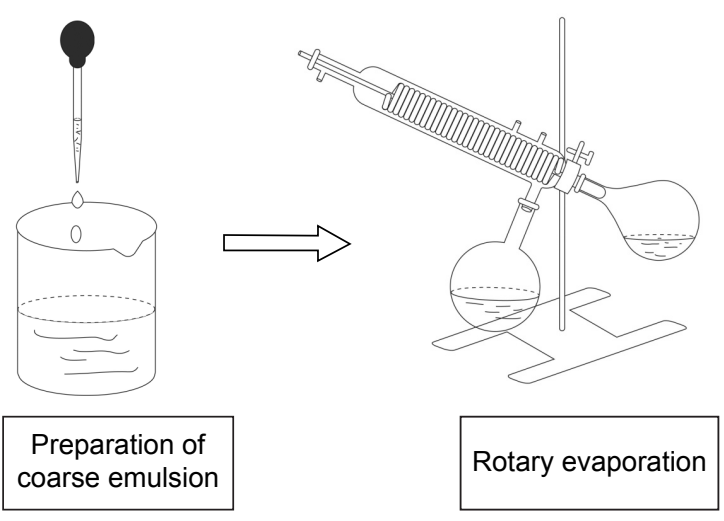

Figure I The structure of rhein and flow chart of preparation of the nanoparticle.

The most widely used class of biocompatible and biodegradable polymers is that of aliphatic polyesters, including poly(D,L-lactic acid), poly( $\varepsilon$-caprolactone), and poly(lacticco-glycolic acid) (PLGA). ${ }^{18}$ Among them, PLGA copolymers have been developed in the past 10 years. PLGA is approved by the US Food and Drug Administration and European Medicine Agency in various drug delivery systems for humans. Polymers with different molecular weights and copolymer compositions are commercially available. PLGA is a high polymer polymerized by polylactic acid and glycolic acid monomers with different proportions. The polymer degradation time can vary from several months to years, depending on the molecular weight and copolymer ratio. PLGA is nontoxic, nonirritating, and fully biodegradable with good biocompatibility and human adaptability. In vivo, the final degradation product of PLGA is lactate that can be metabolized by intravital cells. The forms of PLGA are usually identified by the monomers ratio used. For example, PLGA 50:50 indicates a copolymer composed of 50\% lactic acid and 50\% glycolic acid. Poly(lactic acid) has been used to a lesser extent than PLGA due to the lower degradation rate. It can be completely degraded to carbon dioxide and water and excreted out of the body. It is safe and will not cause significant inflammatory response, immune response, or cell toxicity. It has the advantage of self-degradation in vivo and can be excreted to avoid secondary damage caused to the patient. It is a biodegradable carrier material with good biocompatibility. ${ }^{19}$

The purpose of this paper was to develop a novel rhein formulation based on PLGA NPs suitable for oral administration. The designed nanosystems were obtained by a modified spontaneous emulsification solvent diffusion method and were characterized in terms of morphology, encapsulation efficiency (EE), in vitro release kinetics, and in vivo pharmacokinetics. Moreover, the antiproliferative efficacy of the most promising nanoparticulate prototype was assessed by in vitro cytotoxicity assay toward a human gastric cancer cell line SGC-7901.

\section{Materials and methods Chemicals and reagents}

Rhein (purity $>99 \%$ ) was supplied by JingRong Biopharm Company (Shanghai, People's Republic of China). PLGA (molecular weight, $\sim 15,000 \mathrm{Da}$, lactide/glycolide ratio, 50/50) was purchased from Daigang Biopharm Co., Ltd (Jinan, People's Republic of China). Poloxamer 188 was purchased from Sinopharm Chemical Reagent Co., Ltd. (Shanghai, People's Republic of China). All other chemicals were of analytical grade. Purified water obtained from a Milli-Q system (EMD Millipore, Billerica, MA, USA) was used throughout the experiment.

\section{Preparation of rhein-PLGA-NPs}

Rhein-PLGA-NPs were prepared according to the classical spontaneous emulsification solvent diffusion method. ${ }^{20}$ The procedure was as follows: $20 \mathrm{mg}$ rhein and $100 \mathrm{mg}$ PLGA were codissolved in $12.5 \mathrm{~mL}$ of mixture organic solvent consisting of acetone/ethanol $(3 / 2, v / v)$. The mixture solution was then added dropwise into $35 \mathrm{~mL}$ of the aqueous solution containing $1 \%(\mathrm{w} / \mathrm{v})$ of poloxamer 188 in a $100 \mathrm{~mL}$ flask with stirring at $300 \mathrm{rpm}$. The coarse emulsion was subjected to $400 \mathrm{~W}$ of ultrasonic treatment for 2 minutes using a high-intensity probe ultrasonicator with water bath $\left(0^{\circ} \mathrm{C}\right)$. In the state of reduced pressure, organic solvent was removed from the dispersion system by a rotary evaporator. The final suspension volume, about $10 \mathrm{~mL}$, was then passed through a $220 \mathrm{~nm}$ mesh sieve to remove aggregates (recovery rate $>90 \%$ ). The prepared NPs were collected by the method of ultracentrifugation $\left(12,000 \mathrm{rpm}, 3\right.$ hours, $\left.4^{\circ} \mathrm{C}\right)$, and washed with distilled water at least three times to remove the surfactant. After subsequent analysis, NPs obtained were freeze dried into white powder. The final product was stored in a vacuum desiccator at room temperature.

\section{HPLC conditions and sample preparation}

The concentration of rhein was determined by high-performance liquid chromatography (HPLC) instrument (Agilent 1100). ${ }^{21}$ Solvent delivery system was equipped with a column heater and a plus autosampler. Detection was taken on an 
Agilent 1100 detector. Chromatographic separations were performed on a reversed phase C18 column $(4.6 \mathrm{~mm} \times 150 \mathrm{~mm}$, $5 \mu \mathrm{m}$, Dikma Diamonsil column). And the column temperature was kept at $30^{\circ} \mathrm{C}$. Methanol $/ 0.1 \%$ phosphoric acid $(75 / 25, v / v)$ was used as mobile phase at a flow rate of $1 \mathrm{~mL} / \mathrm{min}$. Detection wavelength was $254 \mathrm{~nm}$.

Each $200 \mu \mathrm{L}$ aliquot of plasma was spiked with $10 \mu \mathrm{L}$ of internal standard working solution (Mitoxantrone, $2 \mathrm{mg} / \mathrm{mL}$ ). Then, $1 \mathrm{~mL}$ of acetonitrile was added to the sample to precipitate plasma proteins. The mixture was vortex mixed for 30 seconds and centrifuged at 10,000 rpm for 10 minutes. The resulting supernatant was transferred to a glass tube and evaporated to dryness under a nitrogen stream at $45^{\circ} \mathrm{C}$. The residue was reconstituted with $100 \mu \mathrm{L}$ of the mobile phase, vortex mixed for 1 minute, and placed in an ultrasonic bath at room temperature for 1 minute. The reconstituted samples were centrifuged at $12,000 \mathrm{rpm}$ for 5 minutes. The final samples $(20 \mu \mathrm{L})$ were placed in amber autosampler vials for HPLC analysis (about 20 minutes per sample).

\section{Characterization of NPs}

Particle size distribution and zeta potential of NPs were determined by Nano-ZS, Malvern Instruments Ltd (Malvern, UK). The polydispersity index (PDI) values were also provided directly by the same instrument. The temperature was kept at $25^{\circ} \mathrm{C}$ during measuring. And all results were the mean of three test runs.

To observe the morphology of the NPs using a transmission electron microscope (TEM) (H-6009IV, Hitachi, Tokyo, Japan), certain amount of the NPs was diluted with distilled water and placed on copper grid covered with nitrocellulose. Then, phosphotungstic acid was used to negative stain the sample, and finally, the sample was dried at room temperature for observation.

\section{Drug loading and entrapment efficiency}

Rhein-loaded PLGA NPs $(0.25 \mathrm{~mL})$ were introduced into preweighed eppendorf tube and lyophilized to constant weight; the dried deposit was dissolved in $0.1 \mathrm{~mL}$ of dichloromethane and diluted by acetonitrile; the amount of rhein in the solution was determined by HPLC. Drug loading (DL) and EE of drugloaded NPs were calculated according to Equations 1 and 2:

$$
\begin{gathered}
\operatorname{DL}(\%)=W_{\mathrm{M}} /\left(W_{\mathrm{P}}+W_{\mathrm{M}}\right) \times 100 \\
\operatorname{EE~}(\%)=W_{\mathrm{M}} / W_{\mathrm{F}} \times 100
\end{gathered}
$$

where $W_{\mathrm{P}}$ is the weight of initial feeding polymer, $W_{\mathrm{M}}$ is the weight of drug incorporated in NPs, and $W_{\mathrm{F}}$ is the weight of initial feeding drug.

\section{Thermal analysis}

Differential scanning calorimetry (DSC) was performed on rhein, PLGA NPs, rhein and PLGA NPs mix, and rheinloaded PLGA NPs. For the structural, crystal, and physical state characterization of drug, DSC studies were performed. The DSC measurements were performed on a Shimadzu DSC (DSC 60) with thermal analyzer. Accurately weighed samples (about $10 \mathrm{mg}$ ) were placed in a sealed aluminum pan before they were heated under nitrogen flow $(50 \mathrm{~mL} /$ min) at a scanning rate of $10^{\circ} \mathrm{C} / \mathrm{min}$ from $100^{\circ} \mathrm{C}$ to $400^{\circ} \mathrm{C}$. An empty aluminum pan was used as reference.

\section{In vitro release}

In vitro release properties of rhein from the NPs were investigated in an aqueous release medium phosphate-buffered saline (PBS; $0.01 \mathrm{M}, \mathrm{pH} 7.4$, containing 20\% ethanol) by a dialysis method. Two milliliters of rhein suspensions (0.5\% hydroxypropyl methyl cellulose [HPMC]; $2 \mathrm{mg}$ rhein) and NPs (2 mg rhein) were introduced into a dialysis bag (molecular weight cutoff $=14 \mathrm{kDa}$, Green Bird Inc., Shanghai, People's Republic of China). The end-sealed dialysis bag was immersed into $50 \mathrm{~mL}$ of release medium at $37^{\circ} \mathrm{C}$, which was stirred at a speed of $60 \mathrm{rpm}$ for 24 hours. Samples of $2 \mathrm{~mL}$ were withdrawn at different time intervals ( 0.5 hour, 1 hour, 2 hours, 3 hours, 4 hours, 6 hours, 8 hours, 10 hours, 12 hours, 14 hours, 16 hours, and 24 hours) and replaced with an equal volume of fresh release medium. The concentration of rhein in the samples was determined by the HPLC method.

\section{Pharmacokinetic study}

Twelve Sprague-Dawley rats (purchased from Shanghai Institute of Pharmacology and Toxicology) were used to investigate the effect of NPs formulation on the pharmacokinetics of rhein after oral administration. The animals were kept in a well-ventilated animal room. The temperature was maintained at $23^{\circ} \mathrm{C} \pm 2{ }^{\circ} \mathrm{C}$ and relative humidity at $50 \% \pm 10 \%$. Throughout the study, the animals had free access to water. This study was conducted following current ethical regulations on animal research of this institute, and all animals used in the experiment received humane care.

Rats were divided into two groups at random and given a single dose of $7.5 \mathrm{mg} / \mathrm{kg}$ of the rhein-loaded PLGA NPs and rhein suspensions $(0.5 \%$ HPMC) by oral administration (1 mL gastric lavage device). Blood samples $(0.5 \mathrm{~mL})$ were collected into heparinized tubes from the orbital vein at 0.25 hour, 0.5 hour, 1 hour, 1.5 hours, 2 hours, 4 hours, 6 hours, 8 hours, 10 hours, 12 hours, and 24 hours after oral administration. Blood was immediately processed for plasma 
by centrifugation at $4,000 \times g$ for 10 minutes. Plasma samples were frozen and maintained at $-70^{\circ} \mathrm{C}$ until analysis.

\section{In vitro cytotoxicity assay}

The cytotoxicity of blank PLGA NPs, free rhein, and rheinloaded PLGA NPs on human gastric cancer cell line SGC-7901 was evaluated by cell proliferation assay. Cancer cell viability of the drug-loaded NPs was evaluated by 3-(4,5-dimethylthiazol-2-yl)-2,5-diphenyltetrazolium bromide (MTT) assay. Human gastric cancer cell line SGC-7901 was purchased from Bioon Biological Pharmaceutical Co., Ltd., and then cultured in Revolutions-Per-Minute Indicator-1640 medium (Hyclone, Logan, UT, USA) supplemented with 10\% heat-inactivated calf serum (Gibco, Grand Island, NY, USA), $100 \mathrm{U} / \mathrm{mL}$ of penicillin, and $100 \mathrm{mg} / \mathrm{mL}$ of streptomycin. The culture environment contained $5 \% \mathrm{CO}_{2}$ and was maintained at constant temperature and humidity of $37^{\circ} \mathrm{C}$. The cells were incubated with free rhein and rhein-loaded PLGA NPs at $0.05 \mu \mathrm{g} / \mathrm{mL}$, $0.5 \mu \mathrm{g} / \mathrm{mL}$, and $5.0 \mu \mathrm{g} / \mathrm{mL}$ equivalent rhein concentrations, blank PLGA NPs, and untreated control group with the same NP concentrations for 24 hours, 48 hours, and 72 hours, respectively. MTT dye was added to each well and incubated at $37^{\circ} \mathrm{C}$ for 4 hours. The supernatant was then removed, and the purple formazan precipitate was dissolved in $150 \mu \mathrm{L}$ of dimethyl sulphoxide. Upon complete dissolution, the absorbance was measured at $490 \mathrm{~nm}$ wavelength. The inhibition effect of rhein on tumor cell growth was measured by the percentage of inhibited cell growth. The measured absorption values should deduct the value of blank media. Survival percentage was calculated as follows: (drug treatment group $[A] /$ control group $[A]$ value $) \times 100$. Each assay was performed in parallel for three times, and the results were expressed as mean \pm standard error of the mean.

\section{Statistical analysis}

Results were expressed as the mean values \pm standard deviation. Statistical analysis was evaluated by two-tailed Student's $t$-test or by two-way analysis of variance followed by Dunnett's $t$-test, with the level of significance chosen at $P<0.05$.

\section{Results and discussion Characterization of NPs}

The preparation method described in this article seems to be suitable for rhein-loaded PLGA NPs. It was carried out by using a polymer concentration in organic phase of $8 \mathrm{mg} / \mathrm{mL}$ and a solvent:poloxamer 188 solution with a ratio of 2:7. Choosing acetonitrile as a solvent was primarily because it can facilitate the dissolution of the polymer and mix with water more easily.

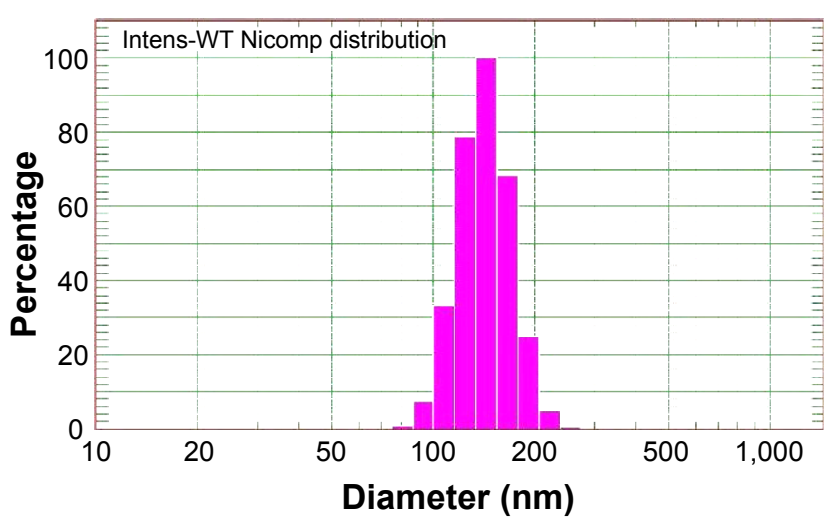

Figure 2 Mean size distribution of rhein-loaded PLGA nanoparticles. Abbreviation: PLGA, poly(lactic-co-glycolic acid).

All NP batches showed a particle diameter ranging from $80 \mathrm{~nm}$ to $210 \mathrm{~nm}$, suitable to obtain an effective intracellular uptake. ${ }^{22}$ Mean size of the NPs after drying was $140.5 \pm 4.3 \mathrm{~nm}$, and the zeta potential of NPs was $-16.9 \pm 3.1 \mathrm{mV}$. As shown in Figure 2, among all the measurement samples, the dispersion of NPs exhibits a unimodal size distribution, while the PDI $<0.1$ also confirmed that the particle was a typical monodisperse system. This indicated that the addition of poloxamer 188 can generate a more homogeneous NP dispersion.

Rhein-loaded PLGA NPs with an average DL of $3.9 \% \pm 0.7 \%$ and $\mathrm{EE}$ of $84.5 \% \pm 6.2 \%$ were obtained with optimized preparation parameters, suggesting that the DL and $\mathrm{EE}$ were high enough for further studies.

In the system, poloxamer 188 had main advantages in the following aspects: (1) Acetone and ethanol can easily diffuse in the aqueous phase with surfactant, and the whole process is done quickly and spontaneously; NPs can easily achieve smaller particle size without adhesions. (2) In the preparation process, ethanol diffused into water with a quick speed; since rhein was insoluble in water but can be dissolved in acetone, PLGA which was more soluble in acetone can continue to slowly encapsulate rhein. Therefore, the resulting NPs had higher EE. It was shown that this method was highly suitable for preparing NPs of insoluble drugs. The amount of DL of the NPs prepared in this experiment is not high, which may be due to the nature of rhein drugs. DL of rhein was influenced by polymer; the longer the polymer chains are, the stronger the hydrophobia is; the higher the compatibility with rhein is, the higher the DL would be. In this experiment, the relative molecular mass of PLGA polymer was 15,000 , leading to low DL amount. If the study chose PLGA with larger relative molecular mass, the particle size of NPs would increase accordingly. This study investigated NPs for oral administration, and small particle size is more conducive to oral absorption. 


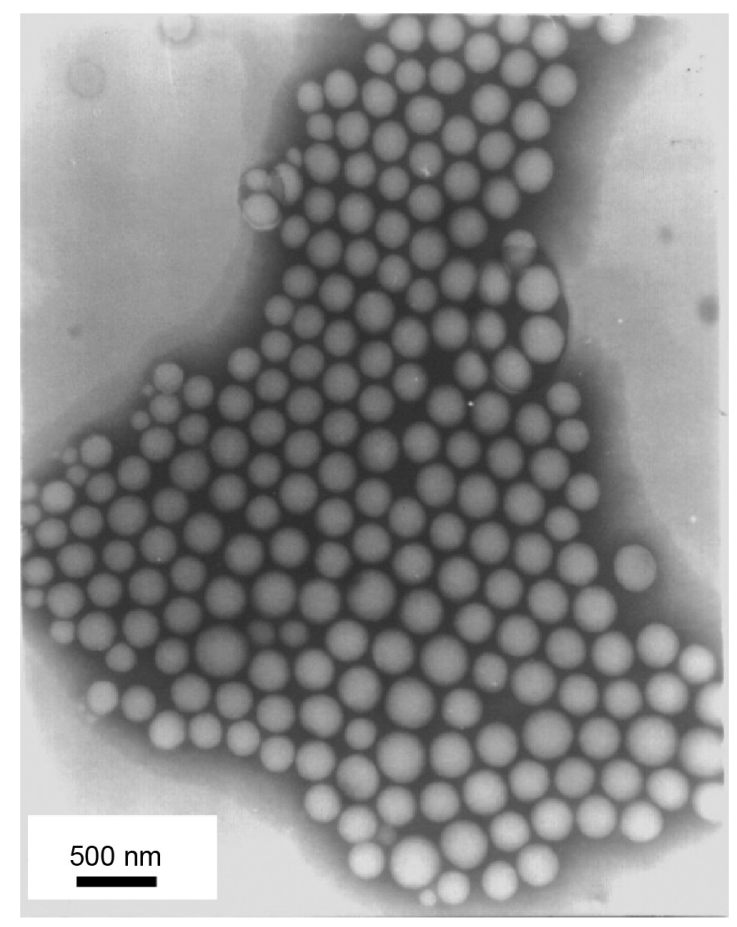

Figure 3 Transmission electron microscopy of rhein-loaded PLGA nanoparticles $(\times 50,000)$.

Abbreviation: PLGA, poly(lactic-co-glycolic acid).

The morphology of rhein-loaded PLGA NPs was investigated by TEM analysis. As seen in Figure 3, the NPs were spherical, had smooth surface, and had no particle aggregation. Partial fusion was detected in some NP samples during the analysis, where many NPs congregate together. DSC thermal analysis was performed on rhein, PLGA NPs, rhein and PLGA NPs mix, and rhein-loaded PLGA NPs. Figure 4 of DSC thermogram shows that rhein had a particular peak at about $321^{\circ} \mathrm{C}$, which also appeared in physical mixture of blank NPs and rhein, indicating that rhein existed as crystal in its natural state and did not have chemical interaction with the blank NPs, whereas the disappearance of the particular peak in rhein-loaded PLGA NPs revealed that rhein existed in PLGA NPs in a form of uncrystallization rather than crystallization just as in physical mixture of blank NPs and rhein.

\section{In vitro release}

The cumulative percentages of rhein released from NPs based on PLGA polymers are reported in Figure 5. As can be seen from the release profile, the release rate of the drug depends on the characteristics of the polymer matrix. Specifically, PLGA-loaded NPs had slow release characteristics (within 5 hours, only about $70 \%$ of rhein was released) which can be attributed to the stronger hydrophobic interaction between hydrophobic domain of PLGA and drug.

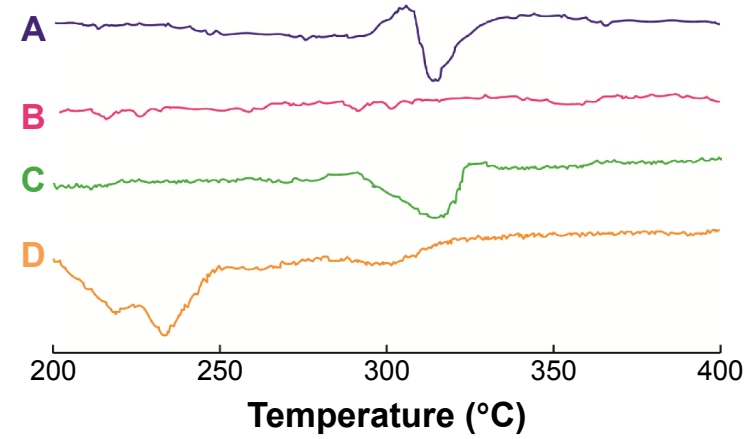

Figure 4 DSC analyses of the samples: (A) rhein, (B) PLGA nanoparticles, (C) physical mixture of rhein and PLGA nanoparticles, and (D) rhein-loaded PLGA nanoparticles.

Abbreviations: DSC, differential scanning calorimetry; PLGA, poly(lactic-coglycolic acid).

In contrast, more than $80 \%$ of drug was released in the first hour of dissolution process in the release medium. Almost $100 \%$ of rhein was released in the first 2 hours. Rhein was gradually released from the NPs as time lapsed, suggesting that rhein was well entrapped in PLGA NPs.

In order to investigate the kinetic modeling of drug release from NPs, the dissolution profiles were fitted to zero order $\left(Q=k_{0} \cdot t\right)$, first order $\left(\ln (100-Q)=\ln Q_{0}-k_{1} \cdot t\right)$, Higuchi $\left(Q=k_{\mathrm{H}} \cdot t_{1 / 2}\right)$, and Korsmeyer-Peppas models. ${ }^{23}$ The model that fitted best for rhein released from the NPs was Higuchi kinetic model with correlation coefficient $r=0.9993$, revealing that rhein release from the NPs could be controlled.

Since rhein has very low solubility in water and the choice of general release media does not meet the requirement of the leakage tank conditions, this study chose pH 7.4 PBS buffer with $20 \%$ ethanol as the release medium, and the release effect was obvious. In addition, as can be seen from the release curve (Figure 5), rhein NPs release showed a twophase state with quick early release rate which significantly slowed down after 3 hours. The cause may be the early-stage quick release of drug on NP surface or in shallow region. The slower release at later stage may be attributed to slower diffusion of drug.

\section{In vivo studies}

The study validated HPLC method for analysis. The results showed that in the studied concentration range, the linear measurement results satisfied the demand, and the impurities did not interfere with the determination of endogenous drugs. Plasma concentration-time profiles of rhein after oral administration of NPs and suspensions are shown in Figure 6. Pharmacokinetic parameters are shown in Table 1. As shown in Figure 6, drug plasma concentration reached a higher level (more than $6 \mu \mathrm{g} / \mathrm{mL}$ ) in the initial 1 hour after oral 


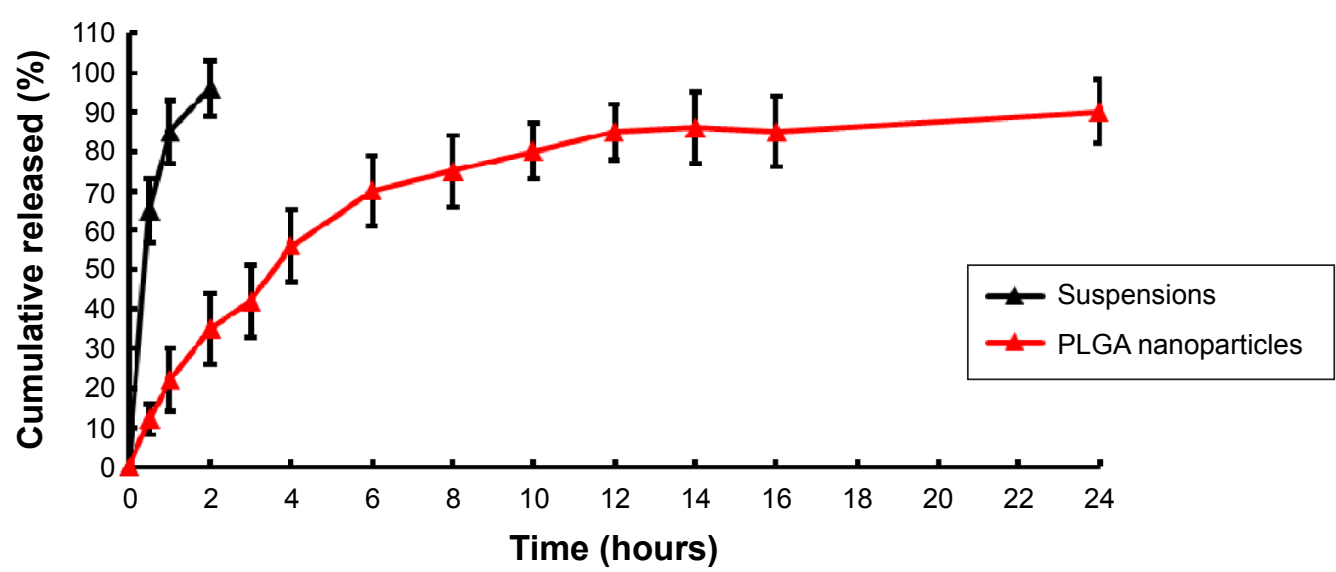

Figure 5 In vitro release of rhein from rhein-loaded PLGA nanoparticles and rhein suspensions. Number represents the percentage of release ( $\mathrm{n}=3$ ). Abbreviation: PLGA, poly(lactic-co-glycolic acid).

administration of rhein suspensions. This was correspondent with initial burst release of drug from suspensions. Then, drug concentration decreased markedly after 2 hours and stayed at a lower level of $0.2-3.9 \mu \mathrm{g} / \mathrm{mL}$. Drug plasma was even lower to show limitation after 10 hours. In contrast, NPs altered the distribution of rhein in vivo, and the half-life after oral administration was prolonged remarkably more than that of suspensions (22.6 hours vs 4.3 hours). The result indicated that NPs had sustained-release efficacy. The area under the curve (AUC) $)_{0-\infty}$ of the NPs formulation was 3.07-fold higher than that of suspensions, suggesting that the encapsulated rhein had mostly been absorbed in rats over the period of 12 hours.

Pharmacokinetically, the NPs system significantly prolonged the elimination half-life, increased the in vivo time of rhein, and thereby enhanced the oral bioavailability. In addition, when NPs were taken up phagocytically, due to the wrapping of the polylactic acid skeleton, rhein can avoid rapid metabolism of CYP oxidase and lower the degradation of PLGA, resulting in sustained-release effect. The improvement of the oral bioavailability was mainly due to the following reasons: (1) The gastrointestinal tract may be bioadhesive, and NPs, small in size, could reside in the gap between the villi, increasing the contact area and residence time in the gastrointestinal tract, so that the body had more time to absorb the drug, thus improving oral absorption and improved bioavailability. (2) The main site of absorption after oral administration of the NPs was in the Peyer's patches where the functional cells were microfold cells (M cells). M phagocytosis opened an ideal channel on intestinal epithelial barrier and constituted the main physiological pathways for NPs non-receptor transport, thus effectively improving the NP absorption in the gastrointestinal tract.

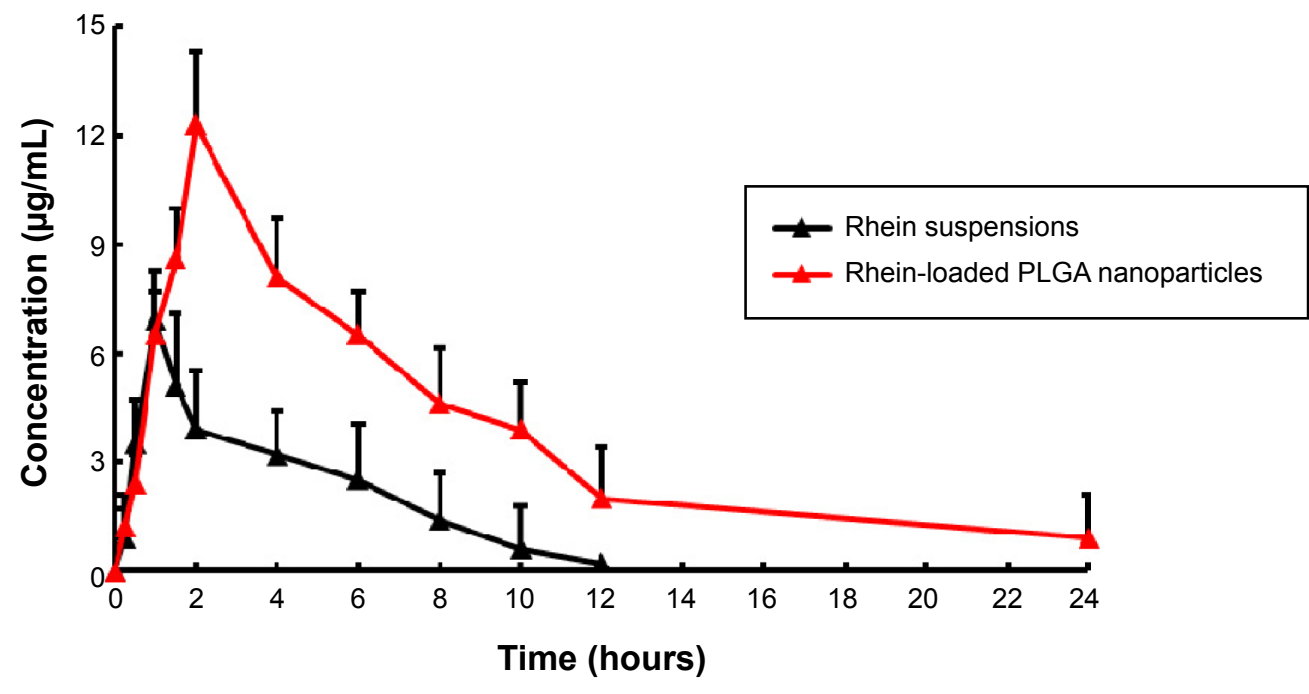

Figure 6 Plasma concentration-time profiles of rhein after oral administration of loaded PLGA nanoparticles and suspensions to rats ( $\mathrm{n}=6$ ). Abbreviation: PLGA, poly(lactic-co-glycolic acid). 
Table I Pharmacokinetic parameters of rhein after oral administration of loaded PLGA nanoparticles and suspensions to rats $(n=6)$

\begin{tabular}{|c|c|c|}
\hline \multirow[t]{2}{*}{ Parameter } & \multicolumn{2}{|l|}{ Oral } \\
\hline & Suspensions & Nanoparticles \\
\hline$T_{\max }$ (hours) & $1.1 \pm 0.3$ & $4.3 \pm 0.5^{*}$ \\
\hline$t_{1 / 2} \alpha$ (hours) & $0.4 \pm 0.1$ & $1.8 \pm 0.4^{*}$ \\
\hline$t_{1 / 2} \beta$ (hours) & $4.3 \pm 0.5$ & $22.6 \pm 2.7^{* *}$ \\
\hline$C_{\max }(\mu g / \mathrm{mL})$ & $6.9 \pm 2.5$ & $12.3 \pm 3.3 *$ \\
\hline $\mathrm{AUC}_{0-t}(\mu \mathrm{g}$ hours $/ \mathrm{mL})$ & $29.2 \pm 6.5$ & $89.7 \pm 9.4 * *$ \\
\hline $\mathrm{AUC}_{0-\infty}(\mu \mathrm{g}$ hours $/ \mathrm{mL})$ & $36.4 \pm 8.7$ & $106.4 \pm 11.4 * *$ \\
\hline MRT (hours) & $4.6 \pm 1.3$ & $8.6 \pm 2.1^{*}$ \\
\hline$C L(L / h)$ & $13.3 \pm 2.6$ & $4.9 \pm 1.4 *$ \\
\hline
\end{tabular}

Notes: $* P<0.05, * * P<0.01$ : rhein-loaded PLGA nanoparticles and rhein suspensions. Abbreviations: PLGA, poly(lactic-co-glycolic acid); $T_{m x}$, peak concentration time; $t_{1 / 2} \alpha$, initial-phase half-life; $t_{1 / 2} \beta$, terminal-phase half-life; $\mathrm{C}_{\max }$, concentration maximum; $A \cup C_{0-t}$, area under the concentration-time curve (from 0 to $t$ time); $\mathrm{AUC}_{0-\infty}$, area under the concentration-time curve (from 0 to $\infty$ time); MRT, mean residence time; $\mathrm{CL}$, clearance.

\section{In vitro cytotoxicity assay}

The in vitro cytotoxicity of rhein both as free drug and loaded into PLGA NPs, at the same drug equivalent concentration of $0.05 \mu \mathrm{g} / \mathrm{mL}, 0.5 \mu \mathrm{g} / \mathrm{mL}$, and $5.0 \mu \mathrm{g} / \mathrm{mL}$, was evaluated by the MTT assay using SGC-7901 as model human gastric cell line. With the same method, the blank PLGA NPs prepared by the same concentration of polymer were evaluated as a control preparation. Figure 7 shows the viability of SGC-7901 cancer cells, cultured with blank PLGA NPs and rhein-loaded PLGA NPs, after incubation for 24 hours (Figure 7A), 48 hours (Figure 7B), and 72 hours (Figure 7C) in comparison with that of pure drug at $0.05 \mu \mathrm{g} / \mathrm{mL}, 0.5 \mu \mathrm{g} / \mathrm{mL}$, and $5.0 \mu \mathrm{g} / \mathrm{mL}$ equivalent rhein concentrations. Since the detected blank PLGA NPs had no significant effect on cell viability, even at high concentrations of PLGA, the NPs of SGC-7901 cells still had no toxic effects, thus demonstrating that the PLGA polymer has good biocompatibility.

In summary, the results clearly showed that, compared to free rhein, rhein-loaded PLGA NPs had better result in inhibiting the growth of tumor cells. Rhein's effect in this regard depends on the dose concentration and incubation time. In particular, after 24 hours of incubation, the SGC7901 cell viability was decreased to about $61 \%, 52 \%$, and $42 \%$ for rhein-loaded PLGA NPs at $0.05 \mu \mathrm{g} / \mathrm{mL}, 0.5 \mu \mathrm{g} / \mathrm{mL}$, and $5.0 \mu \mathrm{g} / \mathrm{mL}$ drug concentration, respectively, corresponding to an increase in cytotoxicity of $22 \%-30 \%(P<0.05)$ compared with that of free rhein. After 48 hours of incubation, SGC-7901 cell viability was decreased to about $51 \%, 40 \%$, and $38 \%$ for the rhein-loaded PLGA NPs and $75 \%, 71 \%$, and $59 \%$ for free drug. More marked inhibition of cell growth was obtained for longer incubation period
(72 hours); at all tested rhein concentrations, rhein-loaded PLGA NPs demonstrated a significant $(P<0.05)$ increase of SGC-7901 growth inhibition with respect to free drug. With an approximately $79 \%$ cell viability reduction, the strongest cytotoxic effect was achieved with NPs at $5.0 \mu \mathrm{g} / \mathrm{mL}$ rhein concentration.

In this study, we observed a dose- and time-dependent antiproliferation effect of rhein-loaded PLGA NPs on these cells. According to research data, studies showed that by inhibiting cyclin D3, CDK4, and CDK6, rhein induced cell cycle arrest of G1/S and G0/G1 and thus ultimately improved the efficacy of cancer chemotherapy. ${ }^{12}$ Other studies have shown that by regulating the activation of nuclear factor kappa B pathway and reducing the expression of vascular endothelial growth factor, rhein can inhibit the expression of matrix metalloproteinase-2 (MMP-2) and MMP-9. This mechanism prompted that rhein had inhibition effect on migration and invasion of tumor cells., ${ }^{8,24}$ The study found that after the drug was formulated into polymer NPs, its cytotoxicity was significantly increased. The reasons were many fold. In fact, when the NPs are adsorbed on the surface of the cell, the drug concentration in the vicinity of the cell membrane can be significantly improved. Thereby, a concentration gradient of the drug both inside and outside the cell membrane was formed and allowed the drug influx into the cells. ${ }^{25}$ The antitumor effect of rhein-loaded PLGA NPs on gastric cancer in vivo and the possible underlying molecular mechanism require further study, while rhein has the potential to be developed as a chemotherapeutic or adjuvant agent for human gastric cancer.

\section{Conclusion}

In this study, a modified spontaneous emulsification solvent diffusion method was used to prepare a novel rhein formulation based on PLGA NPs. The morphology of NPs showed a spherical shape with a smooth surface, without any particle aggregation. Mean size of the NPs was $140.5 \pm 4.3 \mathrm{~nm}$, the average DL was $3.9 \% \pm 0.7 \%$, and EE was $84.5 \% \pm 6.2 \%$.

In vitro and in vivo studies showed that the NPs are characterized by slower release and that they altered the distribution of rhein and remarkably prolonged the half-life after oral administration compared with those of suspensions (22.6 hours vs 4.3 hours). The $\mathrm{AUC}_{0-\infty}$ of the NPs formulation was 3.07-fold higher than that of suspensions. In the in vitro cytotoxicity assay, we observed a dose- and timedependent antiproliferation effect of rhein-loaded PLGA NPs on SGC-7901 cells. Therefore, rhein has the potential 
A

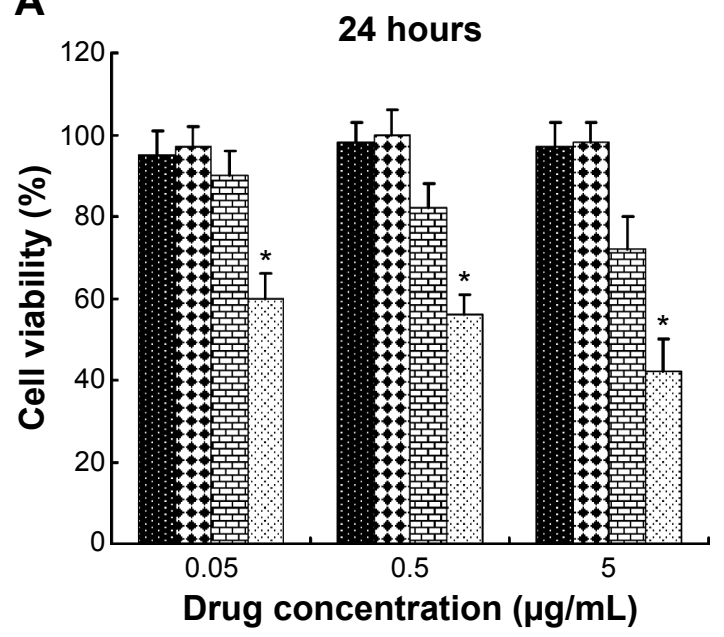

B

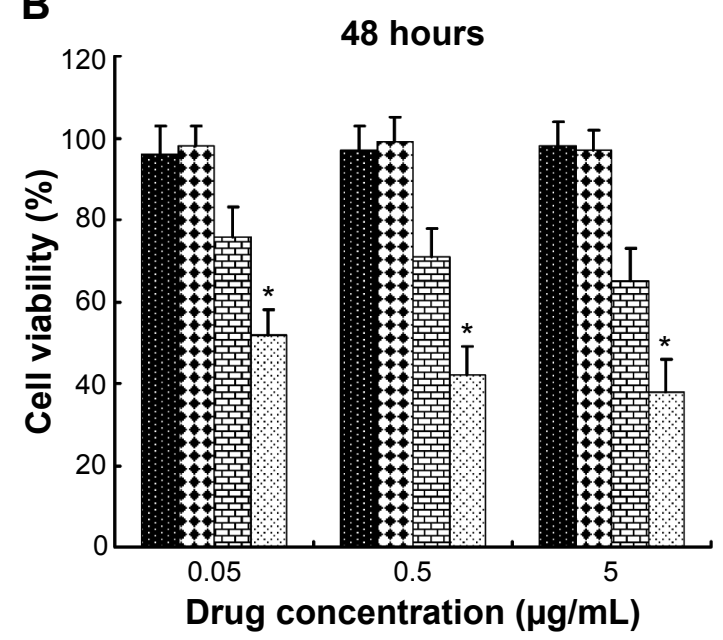

C

72 hours

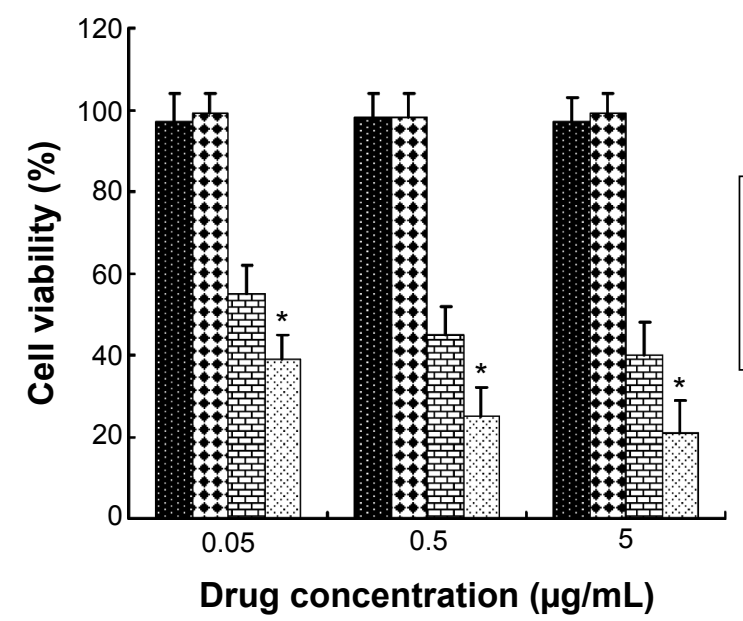

- Untreated control group

- Blank nanoparticles

巴 Rhein (free drug)

$\square$ Rhein-loaded PLGA nanoparticles

Figure 7 Viability of SGC-790I cells cultured with blank nanoparticles, rhein-loaded PLGA nanoparticles after (A) 24 hours, (B) 48 hours, and (C) 72 hours, in comparison with that of pure drug (rhein) at the same dose $(n=3)$.

Notes: $* P<0.05$ : rhein-loaded PLGA nanoparticles and rhein suspensions.

Abbreviation: PLGA, poly(lactic-co-glycolic acid).

to be developed as a chemotherapeutic or adjuvant agent for human gastric cancer.

\section{Disclosure}

The authors report no conflicts of interest in this work.

\section{References}

1. Lin S, Li JJ, Fujii M, Hou DX. Rhein inhibits TPA-induced activator protein-1 activation and cell transformation by blocking the JNKdependent pathway. Int J Oncol. 2003;22(4):829-833.

2. Huang Q, Lu G, Shen HM, Chung MC, Ong CN. Anti-cancer properties of anthraquinones from rhubarb. Med Res Rev. 2007;27(5):609-630.

3. Du Q, Bian XL, Xu XL, Zhu B, Yu B, Zhai Q. Role of mitochondrial permeability transition in human hepatocellular carcinoma Hep-G2 cell death induced by rhein. Fitoterapia. 2013;91:68-73.

4. Blacher E, Ben Baruch B, Levy A, et al. Inhibition of glioma progression by a newly discovered CD38 inhibitor. Int J Cancer. 2015;136(6): $1422-1433$.
5. Castiglione S, Fanciulli M, Bruno T, et al. Rhein inhibits glucose uptake in Ehrlich ascites tumor cells by alteration of membrane-associated functions. Anticancer Drugs. 1993;4(3):407-414.

6. Lai WW, Yang JS, Lai KC, et al. Rhein induced apoptosis through the endoplasmic reticulum stress, caspase- and mitochondria-dependent pathways in SCC-4 human tongue squamous cancer cells. In Vivo. 2009; 23(2):309-316.

7. Chen YY, Chiang SY, Lin JG, et al. Emodin, aloe-emodin and rhein induced DNA damage and inhibited DNA repair gene expression in SCC-4 human tongue cancer cells. Anticancer Res. 2010;30(3): 945-951.

8. Chen YY, Chiang SY, Lin JG, et al. Emodin, aloe-emodin and rhein inhibit migration and invasion in human tongue cancer SCC-4 cells through the inhibition of gene expression of matrix metalloproteinase-9. Int J Oncol. 2010;36(5):1113-1120.

9. Aviello G, Rowland I, Gill CI, et al. Anti-proliferative effect of rhein, an anthraquinone isolated from cassia species, on caco-2 human adenocarcinoma cells. J Cell Mol Med. 2010;14(7):2006-2014.

10. Lin YJ, Zhen YS. Rhein lysinate suppresses the growth of breast cancer cells and potentiates the inhibitory effect of taxol in athymic mice. Anticancer Drugs. 2009;20(1):65-72. 
11. Lin ML, Chung JG, Lu YC, Yang CY, Chen SS. Rhein inhibits invasion and migration of human nasopharyngeal carcinoma cells in vitro by down-regulation of matrix metalloproteinases- 9 and vascular endothelial growth factor. Oral Oncol. 2009;45(6):531-537.

12. Hsia TC, Yang JS, Chen GW, et al. The roles of endoplasmic reticulum stress and $\mathrm{Ca}^{2+}$ on rhein-induced apoptosis in A-549 human lung cancer cells. Anticancer Res. 2009;29(1):309-318.

13. Shi $P$, Huang Z, Chen G. Rhein induces apoptosis and cell cycle arrest in human hepatocellular carcinoma BEL-7402 cells. Am J Chin Med. 2008; 36(4):805-813.

14. Ip SW, Weng YS, Lin SY, et al. The role of $\mathrm{Ca}^{2+}$ on rhein-induced apoptosis in human cervical cancer Ca Ski cells. Anticancer Res. 2007; 27(1A):379-389.

15. Wagner V, Dullaart A, Bock AK, Zweck A. The emerging nanomedicinelandscape. Nat Biotechnol. 2004;24(10):1211-1217.

16. Ganta S, Devalapally H, Shahiwala A, Amiji M. A review of stimuliresponsive nanocarriers for drug and gene delivery. J Control Release. 2008;126(3):187-204.

17. Service RF. Nanotechnology takes aim at cancer. Science. 2005; 310(5751):1132-1134.

18. Musumeci T, Ventura CA, Giannone I, et al. PLA/PLGA nanoparticles for sustained release of docetaxel. Int J Pharm. 2006;325(1-2):172-179.
19. Ford Versypt AN, Pack DW, Braatz RD. Mathematical modeling of drug delivery from autocatalytically degradable PLGA microspheres - a review. J Control Release. 2013;165(1):29-37.

20. Murakami H, Kobayashi M, Takeuchi H, Kawashima Y. Preparation of poly(DL-lactide-co-glycolide) nanoparticles by modified spontaneous emulsification solvent diffusion method. Int J Pharm. 1999; 187(2):143-152.

21. Duan Y, Ji M, Lu X. Simultaneous high-performance liquid chromatographic determination of a rhein-diclofenac prodrug and its active compounds. Pharmazie. 2013;68(1):12-14.

22. Cho K, Wang X, Nie S, Chen Z, Shin DM. Therapeutic nanoparticles for drug delivery in cancer. Clin Cancer Res. 2008;14(5):1310-1316.

23. Mehta AK, Yadav KS, Sawant KK. Nimodipine loaded PLGA nanoparticles: formulation optimization using factorial design, characterization and in vitro evaluation. Curr Drug Deliv. 2007;4(3):185-193.

24. Lin ML, Chung JG, Lu YC, Yang CY, Chen SS. Rhein inhibits invasion and migration of human nasopharyngeal carcinoma cells in vitro by down-regulation of matrix metalloproteinases- 9 and vascular endothelial growth factor. Oral Oncol. 2009;45(6):531-537.

25. Fonseca C, Simoes S, Gaspar R. Paclitaxel-loaded PLGA nanoparticles: preparation, physicochemical characterization and in vitro anti-tumoral activity. J Control Release. 2002;83(2):273-286.
Drug Design, Development and Therapy

\section{Publish your work in this journal}

Drug Design, Development and Therapy is an international, peerreviewed open-access journal that spans the spectrum of drug design and development through to clinical applications. Clinical outcomes, patient safety, and programs for the development and effective, safe, and sustained use of medicines are a feature of the journal, which

\section{Dovepress}

has also been accepted for indexing on PubMed Central. The manuscript management system is completely online and includes a very quick and fair peer-review system, which is all easy to use. Visit http://www.dovepress.com/testimonials.php to read real quotes from published authors.

Submit your manuscript here: http://www.dovepress.com/drug-design-development-and-therapy-journal 